\title{
LHCb: first results
}

\section{Victor Egorychev* for the LHCb Collaboration}

Institute for Theoretical and Experimental Physics (ITEP), Moscow, Russia

E-mail: Victor.Egorychev@cern.ch

The LHCb experiment has been taking data since the LHC startup. Using the few nb ${ }^{-1}$ of already collected data, we show the results on strangeness production, measurement of $b \bar{b}$ production cross-section and the preliminary result on the measurement of the $J / \psi$ production cross section.

The XIXth International Workshop on High Energy Physics and Quantum Field Theory, QFTHEP2010 September 08-15, 2010

Golitsyno, Moscow, Russia

\footnotetext{
*Speaker.
} 


\section{Introduction}

The LHCb experiment is a forward magnetic spectrometer with a polar angle coverage of 15$300 \mathrm{mrad}$ and a pseudo-rapidity range of $1.9<\eta<4.9$ [1]. In order to maximize the probability of a single interaction per bunch crossing, it was designed with an intended limit on the luminosity in the LHCb interaction region to $\sim 2 \times 10^{32} \mathrm{~cm}^{-2} \mathrm{~s}^{-1}$. This has the additional advantage of limiting the radiation damage due to the high particle flux at small angles. The detector consists of a silicon vertex locator, followed by a first Ring Imaging Cherenkov Counter (RICH), a silicon trigger tracker, a 4 Tm spectrometer dipole magnet, tracking chambers, a RICH2 detector, a calorimeter system and a muon identifier. One of the main features is a versatile trigger with a $2 \mathrm{kHz}$ output rate dominated by $p p \rightarrow b \bar{b} X$ events.

A nominal year $\left(10^{7} \mathrm{~s}\right)$ of running in design conditions will give an integrated luminosity of $2 \mathrm{fb}^{-1}$ at $\sqrt{s}=14 \mathrm{TeV}$. However, in the first LHC physics run which started in 2010 the centerof-mass energy is $\sqrt{s}=7 \mathrm{TeV}$. In such conditions, the expected $b \bar{b}$ and $c \bar{c}$ production rates reduce by factor of around 2.3 and 1.8, respectively. In the first few months of operation and machine commissioning, the luminosity has been well below the nominal value. This lower luminosity period allows for lower trigger thresholds for $B$ hadronic decays and also provides a good opportunity to collect rapidly very large samples of charm events. The total integrated luminosity at the time of this conference (September 2010) amounts to $3.2 \mathrm{pb}^{-1}$. Using data collected first results have been obtained on strangeness production and measurement of $b \bar{b}$ production cross-section $(\sigma(p p \rightarrow b \bar{b} X))$ and the preliminary result on the measurement of the $J / \psi$ production cross section.

\section{Results on strangeness production}

Strangeness production provides a sensitive test of soft hadronic interactions. In particular the measurement of strangeness production in hadronic interactions provides input for the understanding of QCD in the non-perturbative regime and for the tuning of Monte Carlo generators.

The production of $K_{s}^{0}$ mesons was studied using data collected with the LHCb detector in $p p$ collisions at $\sqrt{s}=0.9 \mathrm{TeV}$, during the 2009 pilot run of the LHC machine. The integrated luminosity of the analyzed sample was determined using a novel technique, involving measurements of the beam currents, sizes and positions, and was found to be $6.8 \pm 1.0 \mu \mathrm{b}^{-1}$ [2]. The differential prompt $K_{s}^{0}$ production cross section was measured as a function of the $K_{s}^{0}$ transverse momentum $p_{T}$ and rapidity $y$ in the region $0<p_{T}<1.6 \mathrm{GeV} / c$ and $2.5<y<4.0$. As can be seen from Fig. 1, the measurements of the differential cross sections as function of transverse momentum for the three different rapidity bins are in reasonable agreement with the expectations from the PYTHIA generator [3].

The data collected in 2010, both at $\sqrt{s}=0.9 \mathrm{TeV}$ and $\sqrt{s}=7 \mathrm{TeV}$, were also used to study $\Lambda$ production. The preliminary measurements of the $\bar{\Lambda} / \Lambda$ production ratio as a function of the rapidity $y$ for the two center-of-mass energies is presented in Fig. 1.

\section{3. $b-$ production cross section results}

The first data taken with the LHCb experiment at center-of-mass energy $\sqrt{s}=7 \mathrm{TeV}$ have al- 

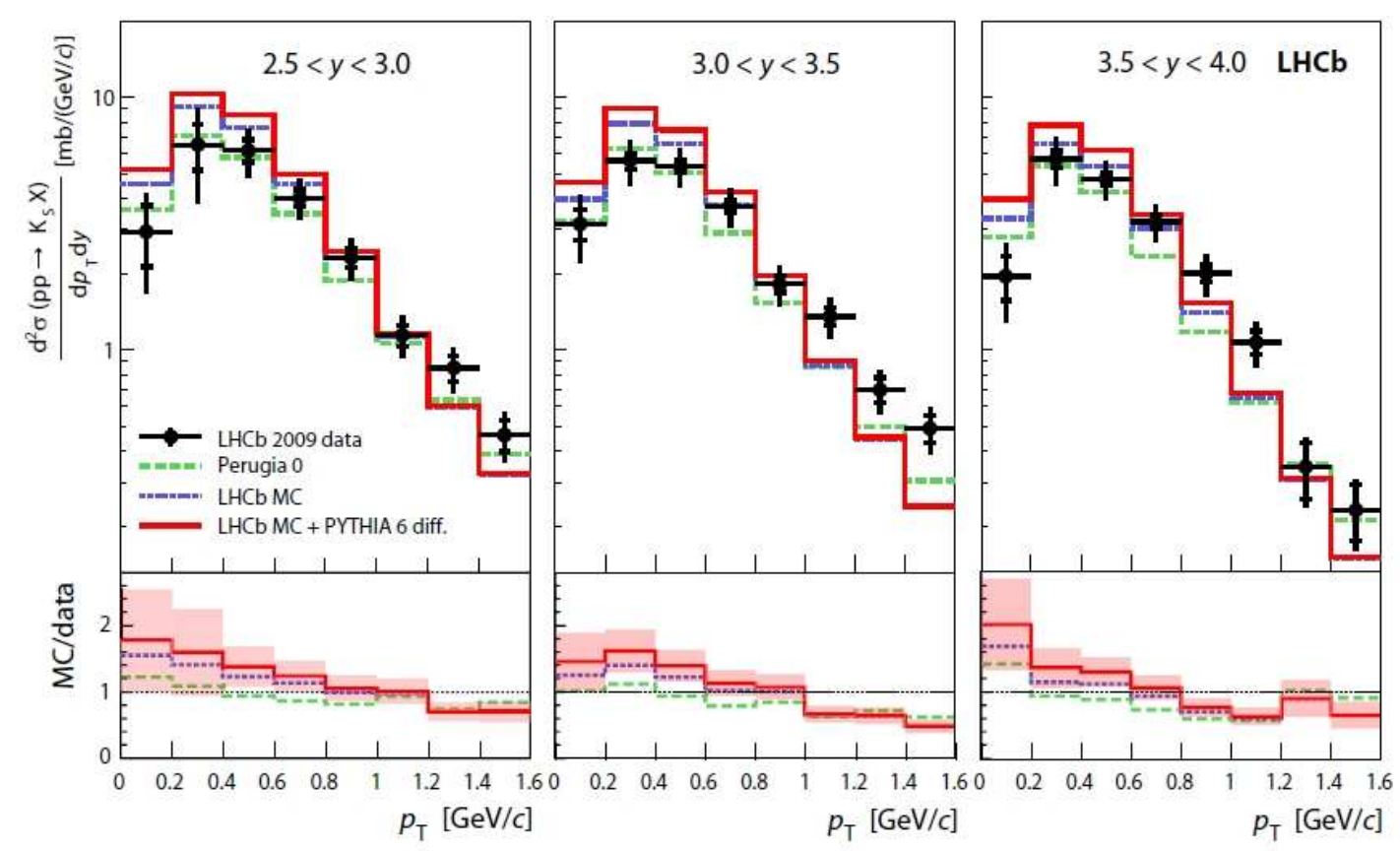

a)

b)
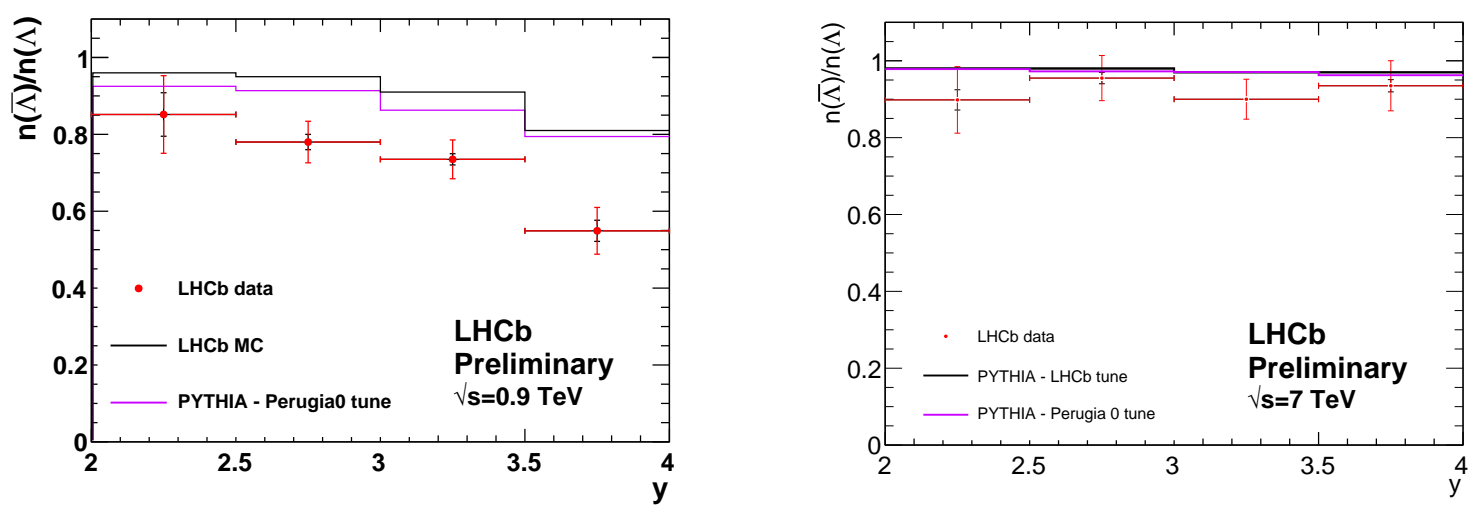

Figure 1: a) Double differential prompt $K_{s}^{0}$ production cross-section in $p p$ collisions at $\sqrt{s}=0.9 \mathrm{TeV}$ as a function of transverse momentum $p_{T}$ and rapidity $y$. b) $\bar{\Lambda} / \Lambda$ production ratio as a function of rapidity $y$ in $p p$ collisions at $\sqrt{s}=0.9 \mathrm{TeV}$ (left) and $\sqrt{s}=7 \mathrm{TeV}$ (right).

lowed the measurement of the $b \bar{b}$ production cross-section. The studies of the cross-section for the production of $b$-flavored hadrons in $p p$ collisions can be used to test the predictive power of QCD. Also, knowledge of the $b$ yield is a critical input in ascertaining the sensitivity of the LHCb experiment in flavor physics. The results of $\sigma(p p \rightarrow b \bar{b} X)$ is based on a sample of $14.2 \mathrm{nb}^{-1}$ collected between April and June 2010 and obtained by two independent methods. The first method [4] uses the inclusive decay $b \rightarrow J / \psi X$. The second method [5] exploits the semileptonic channel $b \rightarrow D^{0} \mu \nu X$, with $D^{0} \rightarrow K \pi$ decay.

\section{$3.1 b \rightarrow J / \psi X$}

The differential production cross section is measured as a function of the $J / \psi$ transverse mo- 
mentum $p_{T}$ in the range $p_{T} \in[0 ; 10] \mathrm{GeV} / c$ and rapidity in the range $y \in[2.5 ; 4]$. The $J / \psi$ mesons are reconstructed in the decay mode $J / \psi \rightarrow \mu^{+} \mu^{-}$and $J / \psi$ from $b$-hadron decays are separated from directly produced (prompt) $J / \psi$ using the $J / \psi$ pseudo-proper time (see below). The acceptance for the $J / \psi$ depends on its polarization. The limited statistics does not allow yet the measurement of the polarization $\alpha$, therefore the cross section has a systematic uncertainty due to the unknown parameter $\alpha$. In Fig. 2 the measured differential cross section is presented for three values of polarization parameter $(\alpha=0, \pm 1)$. The value integrated over the LHCb acceptance is

$$
\sigma\left(\text { inclusive } J / \psi ; p_{T}<10 \mathrm{GeV} / c, 2.5<y<4\right)=7.75 \pm 0.19 \pm 1.10_{-1.27}^{+0.87} \mu \mathrm{b}
$$

where the first error is statistical, the second one systematic and the last error indicates the acceptance uncertainty due to the unknown $J / \psi$ polarization.

To separate the $J / \psi$ produced in $b$ decays the detached vertex analysis is used. The analysis is based on the calculation of the pseudo proper time $t_{z}=d_{z} M / p_{z}$, where $d_{z}$ is the distance along the $z$-axis between the primary vertex and the dimuon vertex, and $M$ and $p_{z}$ are the $J / \psi$ mass and the measured $J / \psi$ momentum in the $z$ direction. The plot of the $t_{z}$ distribution is presented in Fig. 2 . Fitting the exponential decay term yields the fraction of $J / \psi$ from $b$ decays $\left(f_{b}=11.1 \pm 0.8 \%\right.$ ) and a lifetime $\tau_{b}=1.35 \pm 0.10$ ps. From this the cross section for producing a $J / \psi$ from $b$ decay is obtained:

$$
\sigma\left(J / \psi \text { from } b ; p_{T}<10 \mathrm{GeV} / c, 2.5<y<4\right)=0.81 \pm 0.06 \pm 0.13 \mu \mathrm{b}
$$

By extrapolating to the full solid angle using PYTHIA 6.4 [3] the total $b \bar{b}$ production cross section takes the value $\sigma(b \bar{b})=319 \pm 24 \pm 59 \mu \mathrm{b}$, where the first error is statistical and the second one systematic.
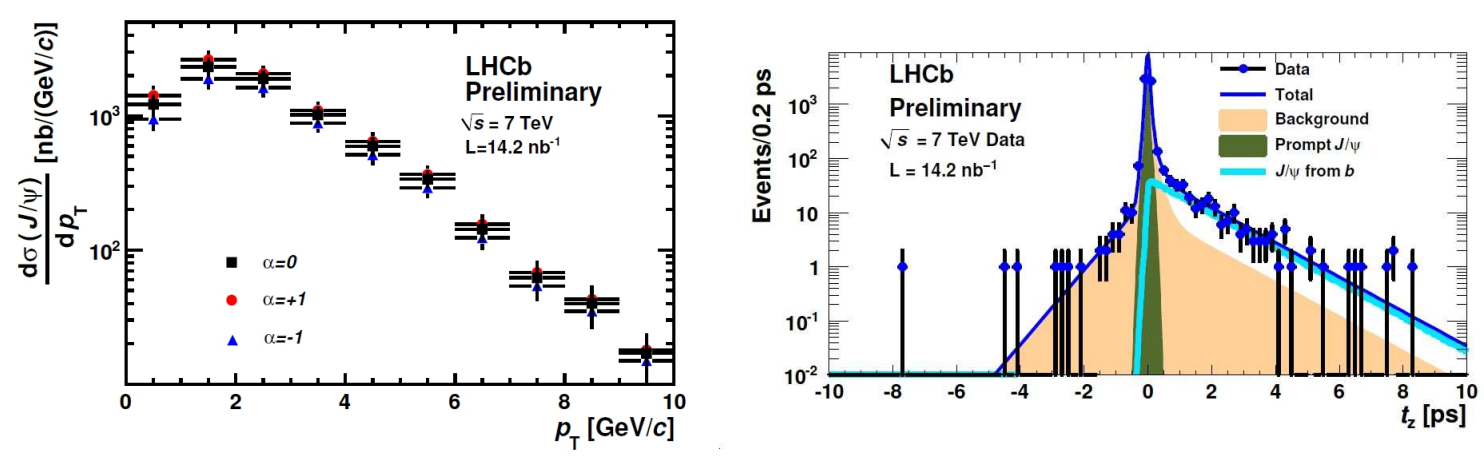

Figure 2: Left: $d \sigma / d p_{T}$ for inclusive $J / \psi$ production, shown for the three representative values of the $J / \psi$ polarization. Right: pseudo proper time (see text) distribution of the $J / \psi$ events, showing the various contributions.

\section{$3.2 b \rightarrow D^{0} \mu \nu X$}

In this analysis the $D^{0}$ mesons produced in $b$ decays are separated from those directly produced by examining the impact parameter (IP) of the reconstructed $D$ track measured with respect to the primary vertex. To reconstruct $D^{0}$ the channel $D^{0} \rightarrow K^{+} \pi^{-}$is used. Full details of the analysis 
are described in [5]. For the measurement two data samples are used. The first was acquired by retaining all interactions where a minimum amount of energy or track hits are observed, called the microbiased sample. The integrated luminosity corresponds to $2.9 \mathrm{nb}^{-1}$. The second sample uses triggers designed to select a single muon, referred to as the triggered sample. Here the integrated luminosity equals $12.2 \mathrm{nb}^{-1}$. The $D^{0} \rightarrow K^{+} \pi^{-}$candidates are vertexed with tracks identified as muons. Signal events from $b$ must have the same sign of the muon and of the kaon (RS). The opposite sign (WS) indicates background events. The IP distribution of both RS and WS candidates, requiring that the $K^{-} \pi^{+}$invariant mass is within $20 \mathrm{MeV} / c^{2}$ of the $D^{0}$ mass, are shown in Fig. 3 confirming that the WS events are highly suppressed.
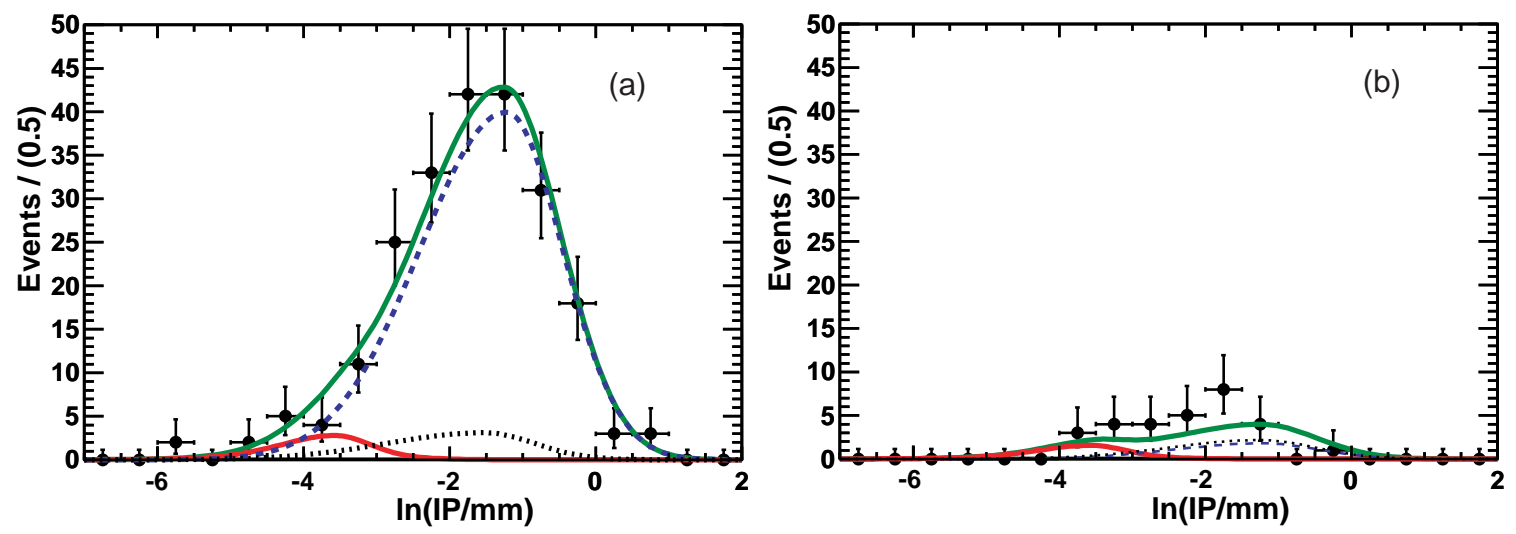

Figure 3: Natural logarithm of the $D^{0}$ IP in the $12.2 \mathrm{nb}^{-1}$ triggered sample for (a) right-sign and (b) wrongsign $D^{0}-\mu$ candidate combinations (see text).

Since the $\theta$ angle of the $b$ hadron is obtained from the $D^{0}-\mu$ vertex, it is possible to measure the differential cross section $d \sigma_{b} / d \eta$. The corresponding results are reported in [5]. Integrating $\sigma$ over the LHCb pseudorapidity acceptance yields the value

$$
\sigma_{b}\left(p p \rightarrow H_{b} X ; 2<\eta<6\right)=75.3 \pm 5.4 \pm 13 \mu \mathrm{b}
$$

Extrapolating to the full solid angle using PYTHIA 6.4 [3] yields the value $\sigma(b \bar{b})=284 \pm$ $20 \pm 49 \mu \mathrm{b}$, in good agreement with the value obtained from the $b \rightarrow J / \psi X$ mode.

\section{Some prospects for fully reconstructed B decays}

A significant mass peak has been obtained by reconstructing the decay $B^{+} \rightarrow D^{0} \pi^{+}$(Fig. 4). This decay is very important proof of principle on the road to the reconstruction of the $B_{s}^{0} \rightarrow D_{s}^{-} \pi^{+}$ signal and the $B \rightarrow D K$ Cabibbo-suppressed decay. The main physics goal with such hadronic $B$ decays is the determination of the CKM angle $\gamma$ using the interference between $b \rightarrow c$ and $b \rightarrow u$ tree-level diagrams in $B_{s} \rightarrow D_{(s)} K$ decays, where a statistical precision of $\sim 7^{\circ}$ is expected with $1 \mathrm{fb}^{-1}$ of data [6].

The current data already allow LHCb to prepare for a few key $B_{s}^{0}$ analyses. The measurement of mixing-induced CP violation in $B_{s}^{0} \rightarrow J / \psi \phi$ decays (Fig. 4) and the search for the very rare $B_{s}^{0} \rightarrow \mu^{+} \mu^{-}$decay based on the first $1 \mathrm{pb}^{-1}$ may reveal possible hints of New Physics [6]. 

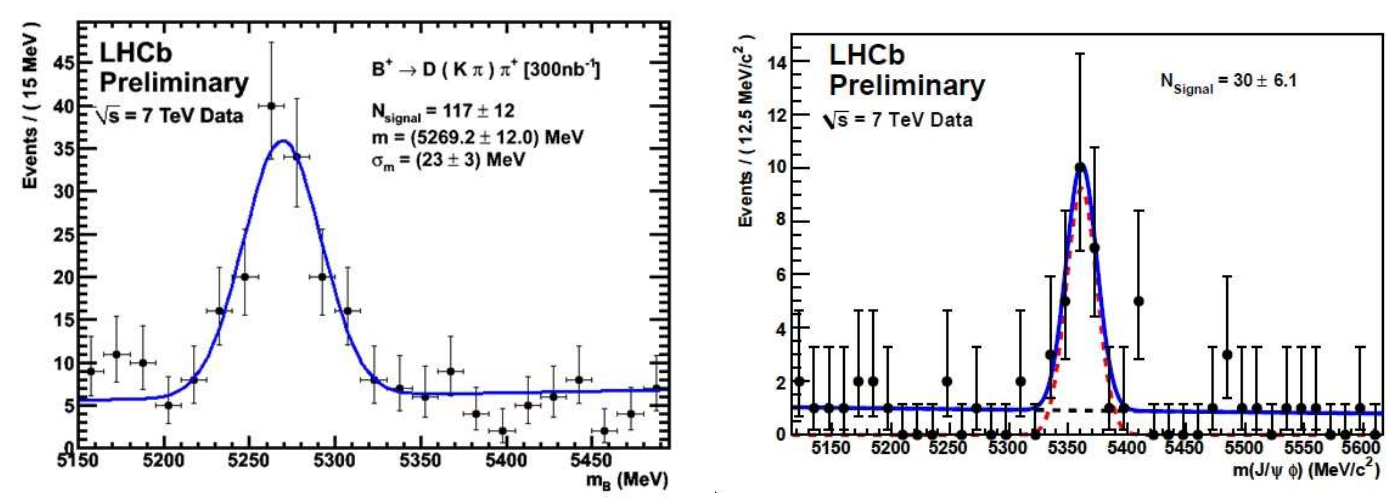

Figure 4: Left: $D^{0} \pi^{+}$invariant mass distribution for $\sim 300 \mathrm{nb}^{-1}$ of data. Right: $J / \psi \phi$ invariant mass distribution for $\sim 600 \mathrm{nb}^{-1}$ of data at $\sqrt{s}=7 \mathrm{TeV}$.

\section{Summary}

The LHCb experiment is in excellent shape and is successfully taking data. First strangeness production measurements have been performed. The $b$-production cross section has been measured in two different channels. The total cross section for inclusive $J / \psi$ production has been obtained. With reasonable assumptions on the integrated luminosity of the 2010-2011 run LHCb will be able to carry out its physics program and access signature sensitive to possible New Physics in CP violation and rare decays.

\section{References}

[1] LHCb Technical Design Report, CERN-LHCC 2003-030.

[2] R.Aaij etal. [LHCb Collaboration], Phys. Lett. B 693 (2010) 69.

[3] T.Sjostrand, S.Mrenna and P.Skands, "PYTHIA 6.4: Physics and manual”, JHEP05 (2006).

[4] LHCb Collaboration, Conference Note CERN-LHCb-CONF-2010-010 (2010).

[5] R.Aaij etal. [LHCb Collaboration], Phys. Lett. B 694 (2010) 209.

[6] B.Adeva etal. [LHCb Collaboration], arXiv:0912.4179v2 [hep-ex]. 